\title{
CryoEM Based Models for Adenovirus Neutralization by Human Alpha-Defensin 5
}

\author{
Neetu Gulati ${ }^{1}$, Jason G. Smith ${ }^{2}$, Glen R. Nemerow ${ }^{3}$, and Phoebe L. Stewart ${ }^{1}$ \\ 1. Department of Pharmacology and Cleveland Center for Membrane and Structural Biology, Case \\ Western Reserve University, Cleveland USA. \\ 2. Department of Microbiology, University of Washington, Seattle USA. \\ 3. Department of Immunology and Microbial Science, The Scripps Research Institute, La Jolla USA.
}

Defensins are peptides of the innate immune system with potent antimicrobial activity. Despite strong interest in defensins as potential pharmaceutical compounds, commercial development of these peptides has been challenging. New generations of modified antimicrobial peptides are being pursued to improve their potential as pharmaceuticals. Strategies include peptide mimetics, hybrid peptides, peptide congeners, stabilized peptides, peptide conjugates and immobilized peptides [1]. Our laboratories have been working on understanding the molecular mechanisms of defensins against adenovirus to characterize key interaction sites. There are six human alpha-defensins, including HD5, and multiple beta-defensins. Defensin action against enveloped viruses includes membrane disruption, as well as interference with viral membrane fusion. Alpha-defensins also neutralize viruses that lack envelopes including human adenovirus (HAdV), human papillomavirus (HPV), and polyomaviruses.

We have used cryo-electron microscopy (cryoEM) to characterize the critical binding site of HD5 on a neutralization-sensitive HAdV vector [2]. In our working structural model HD5 stabilizes the vertex region of the capsid by bridging two capsid proteins, penton base and fiber. This is thought to block further capsid uncoating steps required for infectivity. Viral infectivity studies with virus chimeras comprised of capsid proteins from sensitive and resistant serotypes supported this model [2]. To further describe the critical binding site, we determined subnanometer resolution cryoEM structures of HD5 complexed with both neutralization-sensitive and -resistant HAdV chimeras [3]. CryoEM guided molecular dynamics flexible fitting (MDFF) was used to model the interactions between HD5 and the two chimeras. We found that the long penton base loops of the neutralization-sensitive chimera enveloped HD5 and provided significant stabilization to the vertex region (Fig. 1). It has been shown that HD5 dimerization is important for anti-viral activity against HAdV5 [4].

In this study we analyzed the known sensitivity of multiple HAdV types to HD5 [2] in terms of the emerging structural model for HD5 neutralization of adenovirus. The characteristics of this model are a negatively charged sequence in fiber, represented by 18-EDES-21 in the HAdV35 fiber, and an RGD-containing penton base loop of 39 or more residues with a predicted intrinsically disordered region. The RGD loop length was determined by sequence alignment to the HAdV2 RGD loop (K297-Q374), which is missing due to disorder in the crystal structure (PDB:1X9T). These two characteristics are conserved in multiple adenovirus types shown to be sensitive to neutralization by HD5: adenovirus species B1, B2, C, and E. Adenovirus types resistant to HD5 neutralization differ in their fiber sequence (most have the more hydrophobic GYAR sequence) and have shorter RGD loops (20 to 35 residues). Interestingly, one adenovirus type, species A (type 12), is HD5-sensitive and yet has a short RGD loop of only 15 residues. Modeling of the HAdV12 vertex suggests an alternative binding mode for HD5 (Fig. 2).

We propose that for HAdV12 the short intrinsically disordered RGD loop of penton base is complemented by a longer fiber N-terminal region, which also has predicted intrinsic disorder. 
Modeling indicates that these two intrinsically disordered regions could bracket an HD5 monomer or dimer on two sides. In our alternative model for defensin neutralization of HAdV, HD5 binds to intrinsically disordered regions in two capsid proteins thereby stabilizing the vertex complex of penton base and fiber, and blocking further capsid uncoating steps required for infectivity [5].

\section{References:}

[1] NK Brogden and KA Brogden, Int J Antimicrob Agents 38 (2011) 217.

[2] JG Smith, et al., PLoS Pathog 6 (2010) e1000959.

[3] JW Flatt, et al., PloS One 8 (2013) e61571.

[4] AP Gounder, et al., J Biol Chem 287 (2012) 24554.

[5] The authors acknowledge funding from the NIH National Institute of Allergy and Infectious Diseases (AI042929) to PLS. We thank Dr. JW Flatt for many useful discussions.
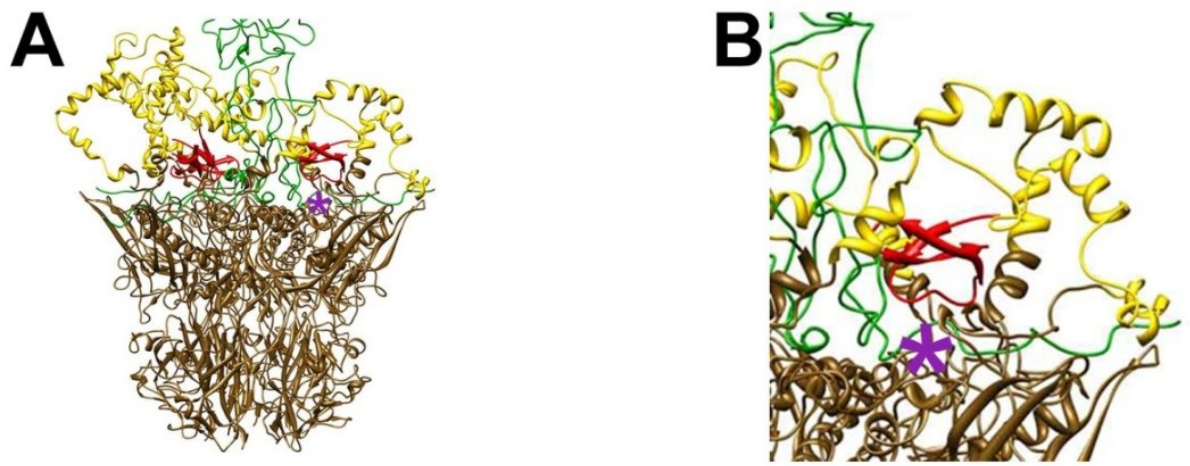

Figure 1. Proposed binding mode for HD5 with the HAdV5 penton of the defensin-sensitive chimera. This model is based on hybrid cryoEM and MDFF results [2,3]. A) Atomic model of the penton base (brown with RGD loops in yellow) and fiber (green) of the chimera with docked HD5 monomers (red). The critical fiber sequence 18-EDES-21 is indicated by an asterisk. B) Enlarged view of one HD5 binding site. HD5 binds above the critical fiber sequence and is enveloped by the RGD loops. HD5 monomers are shown for simplicity, but dimers can be accommodated. Modified from [3].
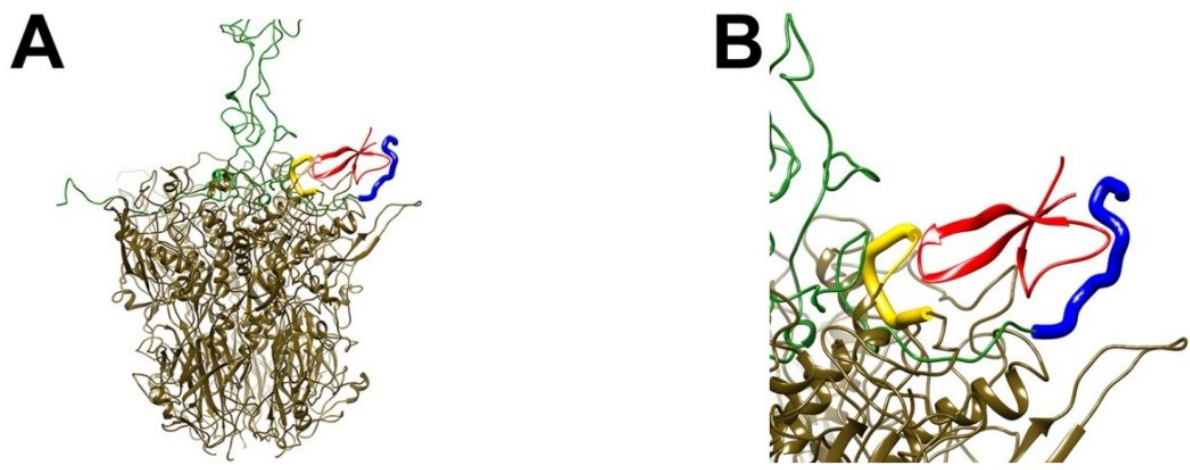

Figure 2. Proposed alternative binding mode for HD5 with HAdV12 penton. A) Atomic model of HAdV12 penton base and fiber with one docked HD5 monomer, colored as in Fig. 1 with the intrinsically disordered region at the N-terminus of the fiber shown in blue. B) Enlarged view of one HD5 binding site. HD5 is bracketed by intrinsically disordered peptide regions (wide tubes) in penton base and the fiber N-terminal region. An HD5 monomer is shown for simplicity, but a dimer can be accommodated. 